\title{
DETECTING WATER BODIES IN LANDSAT8 OLI IMAGE USING DEEP LEARNING
}

\author{
Wei.Jiang ${ }^{1,2}$, Guojin. He ${ }^{1,3,4, *}$, Tengfei Long ${ }^{1,3,4}$, Yuan.Ni ${ }^{1,5}$ \\ ${ }^{1}$ Institute of Remote Sensing and Digital Earth, Chinese Academy of Sciences, Beijing, China-jiangwei@ radi.ac.cn \\ ${ }^{2}$ University of the Chinese Academy of Sciences, Beijing, China \\ ${ }^{3}$ Key Laboratory of Earth Observation Hainan Province, Sanya, China-hegj@ radi.ac.cn \\ ${ }^{4}$ Sanya Institute of Remote Sensing, Sanya, China-longtf@ radi.ac.cn \\ ${ }^{5}$ Key Laboratory of Spatial Data Mining \& Information Sharing of Ministry of Education, Spatial Information Research Center of Fu \\ jian Province, Fuzhou University, Fuzhou, China-269786073@qq.com
}

\section{Commission VI, WG III/5}

KEY WORDS: Water body, Landsat 8, Multi-layer perceptron, Deep learning, Maximum likelihood

\begin{abstract}
:
Water body identifying is critical to climate change, water resources, ecosystem service and hydrological cycle. Multi-layer perceptron(MLP) is the popular and classic method under deep learning framework to detect target and classify image. Therefore, this study adopts this method to identify the water body of Landsat8. To compare the performance of classification, the maximum likelihood and water index are employed for each study area. The classification results are evaluated from accuracy indices and local comparison. Evaluation result shows that multi-layer perceptron(MLP) can achieve better performance than the other two methods. Moreover, the thin water also can be clearly identified by the multi-layer perceptron. The proposed method has the application potential in mapping global scale surface water with multi-source medium-high resolution satellite data.
\end{abstract}

\section{INTRODUCTION}

The spatial and temporal change pattern of the surface water has important practical significance and scientific value for water resources management, biodiversity, emergency response and global climate change(Yamazaki et al., 2015). Remote sensing technology is the efficient and convenient mean for identifying the earth surface water at large scale. A large number of water body extraction methods for various remote sensing data have been proposed over the past few decades. These method can be divided into five categories, namely threshold method, spectral index method, object oriented method and machine learning(Liao et al., 2014), The first one is very simple method to identify the surface water, however, it is difficult to distinguish between surface water and cloud shadows. The spectral index method is the prevalent method for surface water extraction, however, the method cannot remove mountain shadows ice and snow. The object-oriented method usually be used for high resolution remote image, which requires enormous time in image segmentation. The machine learning is to select tremendous training sample and identify surface water using different intelligent algorithm, including maximum likelihood, support vector machine, neural network, deep learning, constraint energy minimization(Ji et al., 2015). These methods are automatic and efficient with less manual work. Recent progress in deep learning has shown the promising solution for the target detection and image classification across the various image processing fields(Guo et al., 2016). Despite the convolutional neural networks under the deep learning architecture has been widely used to identify the target in remote sensing images by using labelled training sample(Isikdogan et al., 2017), few studies have reported the application of deep learning to remote sensing data at large scale. Therefore, this study aims to explore the potential of the multi-layer perceptron based on deep learning framework for identifying water body in Landsat OLI images. Four images covering different water type are selected for assessing the deep learning method by comparing water index method and maximum likelihood.

\section{STUDY AREA AND MATERIALS}

Four regions ranging from coastal to inland in China are selected as the study area. The Landsat8 OLI images corresponding study area are derived from U.S. Geological Survey (https://glovis.usgs.gov/) and the metadata information of these images are showed in Table 1. These images cover different water body type and contain various water noise, including cloud, cloud shadow and mountain shadow.

\begin{tabular}{|c|c|c|c|c|}
\hline NO & Path/row & Time & $\begin{array}{c}\text { Cloud } \\
\text { cover }\end{array}$ & Water type \\
\hline a & $119 / 28$ & $2017-05-02$ & $4.25 \%$ & $\begin{array}{c}\text { River, lake } \\
\text { cluster }\end{array}$ \\
\hline b & $122 / 33$ & $2015-06-12$ & $0.43 \%$ & $\begin{array}{c}\text { sea water, } \\
\text { River, } \\
\text { artificial pond }\end{array}$ \\
\hline c & $122 / 44$ & $2015-10-19$ & $1.12 \%$ & $\begin{array}{c}\text { Water } \\
\text { channel, sea } \\
\text { water }\end{array}$ \\
\hline d & $128 / 40$ & $2017-06-15$ & $2.70 \%$ & River, lake \\
\hline
\end{tabular}

Table 1. Metadata information of the Landsat OLI images

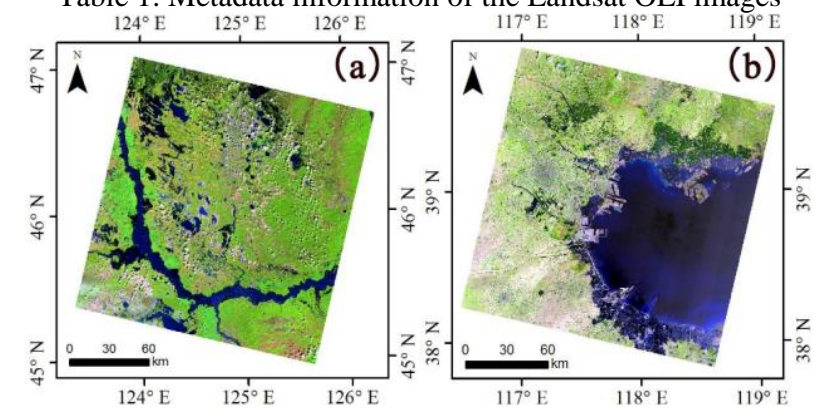

\footnotetext{
* Corresponding author
} 


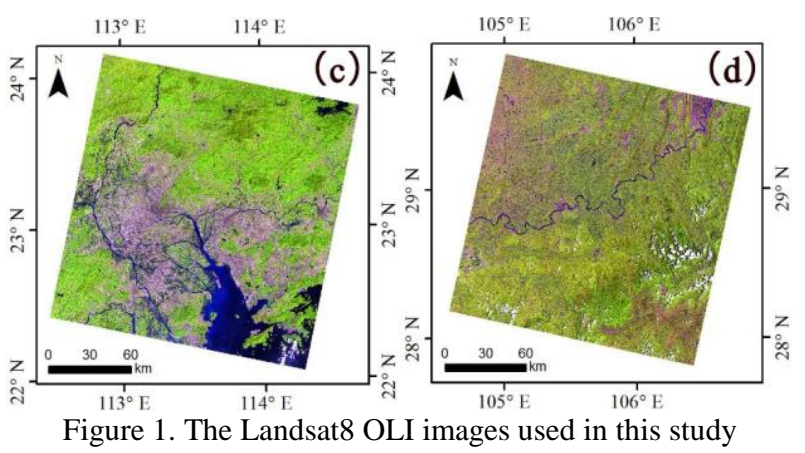

3. METHODOLOGY

\subsection{Image pre-processing}

To obtain high-quality and consistent dataset, download images should be pre-processed. The Landsat 8 image pre-processing consists of radiometric calibration and atmospheric correction. Radiometric calibration need two steps. The first step is to calculate the top of atmosphere (TOA) radiance rTOA using the following equation:

$$
\mathrm{r}_{\mathrm{TOA}}=\mathrm{a} \cdot \mathrm{DN}+\mathrm{b}
$$

DN donates the original digital number (DN) values. The a and $\mathrm{b}$ represent the gain factor and the offset, repressively.

The second step is to calculate the TOA reflectance $\mathrm{R}_{T O A}$, which can correct the error of Earth-Sun distance and exoatmospheric solar irradiance.

$$
\mathrm{R}_{\mathrm{TOA}}=\left(\pi \cdot \mathrm{rTOA} \cdot \mathrm{d}^{2}\right) /(\mathrm{E} 0 \cdot \cos \theta)
$$

d represents Earth-to-Sun distance, E0 donates mean exoatmospheric solar irradiance, $\theta$ is the solar zenith angle and $\pi$ $=3.1415926$.

Furthermore, atmospheric correction is conducted using 6s model with atmospheric parameters (water vapour content, ozone, and aerosol optical thickness), which are derived from MODIS atmospheric level 2 products. Through the above preprocessing, the original images are transformed into surface reflectance.

\subsection{Water extraction algorithm}

\section{(1) Deep learning}

Based on deep learning framework, multi-layer perceptron(MLP) is used in this study to extract surface water. In this model, each neuron in each layer need to be fully connected. Compared with traditional artificial neural network, the more layer is set in multi-layer perceptron to deeply learn the feature of input data(Isikdogan et al., 2017).

Tensorflow is an open source deep learning framework developed by Google and it supports both Python and c ++ programming languages. In this study, multi-layer perceptron with four hidden layers is set. All sample iterates 200 time, and 100 samples per iteration.

(2) Maximum likelihood (ML)

Maximum likelihood is a type of classical supervision classification method based on statistical analysis. The training samples are assumed to be distributed in Gaussian normal distribution, and then are selected for estimating parameter of distribution function(Erbek et al., 2004). The conditional probability density function for $i$ class can be calculated by following equation:
$g_{i}(X)=\ln p\left(\omega_{i}\right)-\frac{1}{2} \ln \left|V_{i}\right|-\frac{1}{2}\left(X-M_{i}\right)^{T} V_{i}^{-1}\left(X-M_{i}\right)(3)$

Where $X$ is the $\mathrm{n}$-dimensional data ( $\mathrm{n}$ is the number of bands), $i$ donates the ith class, $p\left(\omega_{i}\right)$ represents the assume probability for all class. $V_{i}$ is the covariance matrix of class $i$ and $M_{i}$ is the mean vector of a class.

(3) Water index (WI)

Modification of normalized difference water index(MNDWI $)(\mathrm{Xu}, 2006)$ is the most used index to enhance the water information. The equation is as follows:

$$
M N D W I=(B 1-B 2) /(B 1+B 2)
$$

$B 1$ and $B 2$ refer to the Band3 and Band6 of Landsat8 OLI image, respectively. Moreover, Otsu threshold (Xie et al., 2016) method is used to automatically distinguish between water bodies and non-water bodies.

\subsection{Water extraction algorithm}

To evaluate the accuracy of water classification, 200 random points for water and no-water are verified using Google earth high resolution image, respectively. Moreover, the visual analysis will be conducted to compare the classification accuracy.

\section{RESULTS AND DISCCUSSION}

The water and no-water training samples are visually selected on ENVI 5.3 platforms to train the model of multi-layer perceptron and ML. The WI index can automatically identify water without samples. The water classification for three methods are generated, which are showed in Figure2. Overall accuracy and kappa coefficients for each classification map are summarized in Table2 and Table3.

Figure 2 shows the water body classification of three methods for the four experimental regions. With the same training sample, the result using ML and multi-layer perceptron can achieve the similar performance of identifying water body in three regions. The water body mapping results in region $b$ using WI miss the eastern seawater area.

Moreover, the overall accuracy(OA) and kappa coefficients(KCs) of each classification result are calculated to quantitative evaluate classification accuracy, which is showed in table2 and table3. Water body produced by multi-layer perceptron has the highest $\mathrm{OA}$ and $\mathrm{KCs}$ in three methods among different regions. As to region $\mathrm{b}$, the $\mathrm{OA}$ and $\mathrm{KCs}$ of $\mathrm{WI}$ is lower than that of ML and multi-layer perceptron. This quantitative analysis is consistent with figure2 (b1). 

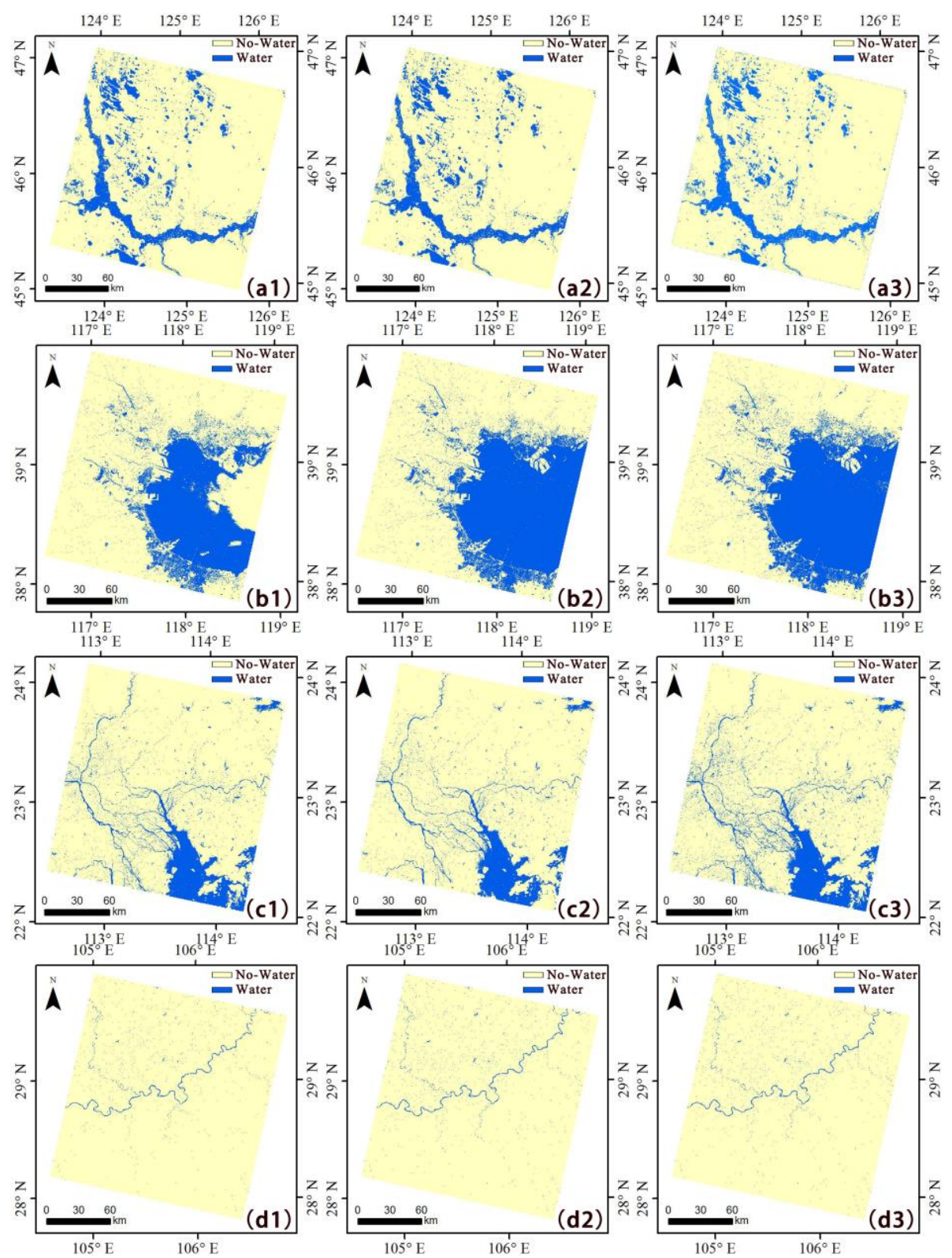

Figure 2. The typical water bodies of Landsat8 OLI (The first column represents water body classification of WI, the second column represents water body classification of ML, the third column represents water body classification of multi-layer perceptron)

\begin{tabular}{|c|c|c|c|}
\hline NO & WI & ML & MLP \\
\hline $\mathrm{a}$ & $98.25 \%$ & $96.50 \%$ & $98.75 \%$ \\
\hline $\mathrm{b}$ & $85.82 \%$ & $98.29 \%$ & $99.27 \%$ \\
\hline $\mathrm{c}$ & $96.75 \%$ & $93.00 \%$ & $99.75 \%$ \\
\hline $\mathrm{d}$ & $90.25 \%$ & $93.50 \%$ & $98.75 \%$ \\
\hline
\end{tabular}

Table 2. Overall accuracy for each classification result

\begin{tabular}{|c|c|c|c|}
\hline NO & WI & ML & MLP \\
\hline $\mathrm{a}$ & 0.965 & 0.930 & 0.975 \\
\hline $\mathrm{b}$ & 0.772 & 0.966 & 0.985 \\
\hline $\mathrm{c}$ & 0.935 & 0.860 & 0.995 \\
\hline $\mathrm{d}$ & 0.805 & 0.870 & 0.975 \\
\hline
\end{tabular}

Table 3. Kappa coefficients for each classification result 
After the quantitative index accuracy classification, the visual inspection of different classifier for four regions is presented in figure 3 . This result shows that multi-layer perceptron achieves better performance of water extraction compared with WI and ML. As to water body classification in region a and region d, ML miss some small ponds and thin river, respectively. Moreover, WI cannot distinguish some seawater from land in region $b$. The comparison shows that multi-layer perceptron can be used to extract the small pond in the aquaculture area with higher accuracy. Therefore, multi-layer perceptron can achieve better performance of water mapping than that of WI and ML by visual assessment.

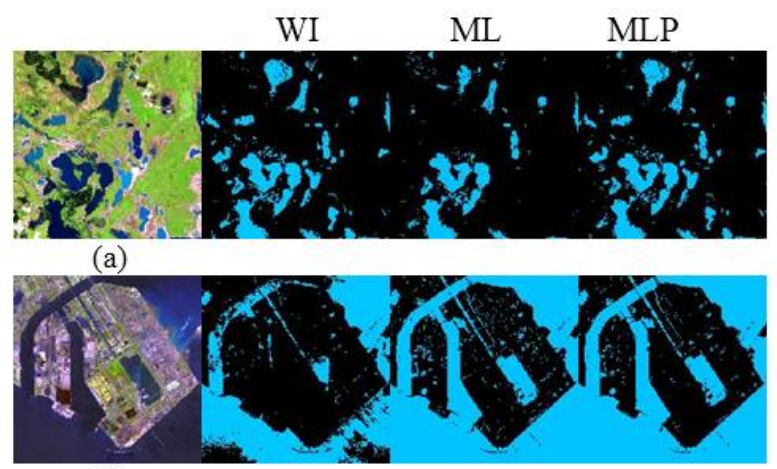

(b)

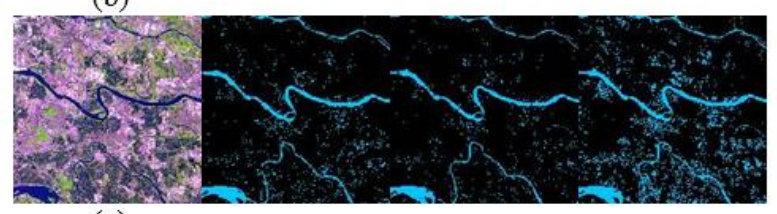

(c)

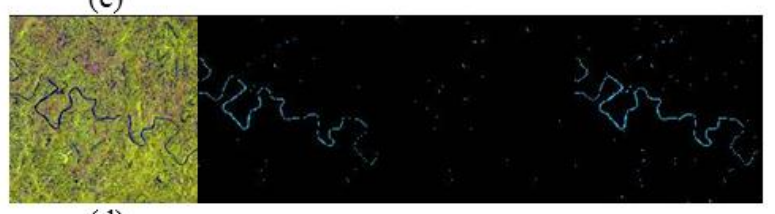

(d)

Figure3 The typical water bodies of Landsat8 OLI

\section{CONCLUSION}

The study adopts multi-layer perceptron to determine the water body of Landsat 8 image. Comparing with ML and WI method, this method is more universal to extract water bodies at different regions. Accuracy indices and local visual comparison demonstrate that multi-layer perceptron has higher classification precision and better performance than that of WI and ML. Moreover, thin water, including small pond and thin river, also can be identified by the multi-layer perceptron. This conclusion shows that multi-layer perceptron can be implemented in mapping large scale surface water in the future.

\section{ACKNOWLEDGEMENTS}

This research is financially supported by the National Key Research and Development Program of China-Rapid production method of large scale global change products (2016YFA0600302), the Hainan Provincial Department of Science and Technology under Grant (Nos.ZDKJ2016021 and ZDKJ2016015) and National Natural Science Foundation of China (61731022 and 61701495).

\section{REFERENCES}

Erbek, F.S., Ozkan, C. and Taberner, M., 2004. Comparison of maximum likelihood classification method with supervised artificial neural network algorithms for land use activities. International Journal of Remote Sensing,25(9), pp.1733-1748.

Guo, Y.M., Liu, Y., Oerlemans, A., Lao, S.Y., Wu, S. and Lew, M.S., 2016. Deep learning for visual understanding: A review. Neurocomputing,187,pp.27-48.

Isikdogan, F., Bovik, A.C. and Passalacqua, P., 2017. Surface Water Mapping by Deep Learning. Ieee Journal of Selected Topics in Applied Earth Observations and Remote Sensing,10(11),pp.17-28.

Ji, L.Y., Geng, X.R., Sun, K., Zhao, Y.C. and Gong, P., 2015. Target Detection Method for Water Mapping Using Landsat 8 OLI/TIRS Imagery. Water,7(2), pp.794-817.

Liao, A.P., Chen, L.J., Chen, J., He, C.Y., Cao, X., Chen, J., Peng, S., Sun, F.D. and Gong, P., 2014. High-resolution remote sensing mapping of global land water. Science China-Earth Sciences,57(10), pp.2305-2316.

Xie, H., Luo, X., Xu, X., Pan, H.Y. and Tong, X.H., 2016. Evaluation of Landsat 8 OLI imagery for unsupervised inland water extraction. International Journal of Remote Sensing,37(8), pp.1826-1844.

Xu, H.Q., 2006. Modification of normalised difference water index (NDWI) to enhance open water features in remotely sensed imagery. International Journal of Remote Sensing,27(14),pp 3025-3033.

Yamazaki, D., Trigg, M.A. and Ikeshima, D., 2015. Development of a global similar to 90 m water body map using multi-temporal Landsat images. Remote Sensing of Environment,171,pp.337-351. 